\title{
TENDENCIA DE LA CESÁREA: UNA REFLEXIÓN DESDE LA ÉTICA
}

\author{
Jairo Javier Vanegas López ${ }^{1}$, Amaya Pávez Lizárraga², Patricia Muñoz Ojeda³
}

Resumen: Las estadísticas mundiales muestran una tendencia al alza en las tasas de cesáreas que superan el 15\% recomendado por la OPS/OMS. Esta cirugía se ha convertido en la más frecuente en los países de ingresos medios y altos. Algunos estudios sugieren que no todas estas cesáreas estarían justificadas. Al respecto se plantean algunas reflexiones sobre los dilemas éticos que se pueden observar desde varias posiciones teóricas, como el consecuencialismo, el kantianismo, la ética de la virtud y la teoría feminista. A su vez, estos dilemas están inmersos en múltiples factores individuales, sociales y culturales, entre otros. Desde la salud pública se debe revisar el parámetro actual definido como "rango aceptable" de cesáreas, ya que puede ser demasiado bajo. Igualmente se recomienda la aplicación de medidas para fortalecer en los pacientes el deseo de un parto normal, cuando sea posible, a través de información y educación oportuna durante la atención prenatal. Las decisiones del profesional de la salud y de las mujeres deben estar respaldadas por la mejor información disponible.

Palabras clave: cesárea, ética, ética de salud pública, mujeres

\section{Cesarean section trend: A reflection from ethics}

\begin{abstract}
World statistics show an upward trend in Cesarean section rates that exceed the $15 \%$ recommended by PAHO / WHO. This surgery has become the most common in high- and middle-income countries. Some studies suggest that not all these caesarean sections would be justified. In this regard, some reflections are made on the ethical dilemmas that can be observed from various theoretical positions such as consequentialism, Kantianism, the ethics of virtue and feminist theory. In turn, these dilemmas are immersed in multiple individuals, social and cultural factors, among others. From Public Health, the current parameter defined as the "acceptable range" of Caesarean sections should be reviewed as it may be too low. It is also recommended that measures be applied to strengthen patients' desire for a normal delivery whenever possible through timely information and education during prenatal care. The decisions of the health professional and women must be supported by the best information available.
\end{abstract}

Keywords: caesarean section, ethics, public health ethics, women

\section{Tendência à cesárea: uma reflexáo a partir da ética}

Resumo: As estatísticas mundiais mostram uma tendência de alta nas taxas de cesáreas que superam em 15\% o recomendado pela OPAS/OMS. Esta cirurgia se converteu na mais frequente em países de renda média e alta. Alguns estudos sugerem que não todas estas cesáreas seriam justificadas. A esse respeito se colocam algumas reflexôes sobre os dilemas éticos que se podem observar desde várias posiciones teóricas, como o consequencialismo, o kantianismo, a ética da virtude e a teoria feminista. Por sua vez, estes dilemas estáo imersos em múltiplos fatores individuais, sociais e culturais, entre outros. Deve-se revisar, a partir da saúde pública, o parâmetro atual definido como "faixa aceitável" de cesáreas, já que pode ser demasiado baixo. Igualmente se recomenda a aplicação de medidas para fortalecer nas pacientes o desejo de um parto normal, quando possível, através de informação e educação oportuna durante a assistência pré-natal. As decisões do profissional da saúde e das mulheres devem estar respaldadas pela melhor informação disponível.

Palavras chave: cesárea, ética, ética de saúde pública, mulheres

\footnotetext{
${ }^{1}$ Facultad de Ciencias Médicas, Escuela Obstetricia, Programa Centro de Salud Pública, Universidad de Santiago de Chile, Santiago, Chile. ORCID: 0000-0001-5281-483X

Correspondencia: jairo.vanegas.1@usach.cl

${ }^{2}$ Facultad de Ciencias Médicas, Escuela Obstetricia, Programa Centro de Salud Pública, Universidad de Santiago de Chile, Santiago, Chile. ORCID: 0000-0002-4671-2092

${ }^{3}$ Facultad de Ciencias Médicas, Escuela Obstetricia y Puericultura. Universidad de Santiago de Chile, Santiago, Chile. ORCID: 0000-00034751-6234
} 


\section{Introducción}

La OPS/OMS ha establecido que la tasa ideal de cesáreas debe oscilar entre el 10\% y el 15\% como procedimiento quirúrgico indicado para mujeres embarazadas. Sin embargo, la frecuencia de este procedimiento quirúrgico está aumentando. Dentro de las principales indicaciones de un procedimiento cesárea se puede mencionar la distocia en el trabajo de parto, sufrimiento fetal agudo, cesáreas previas y presentaciones podálicas. Existen otras situaciones a tomar en cuenta, como la mala interpretación de la monitorización cardíaca fetal, el aumento de intervenciones en edad gestacional menores y el aumento de embarazos en mujeres mayores de 40 ańos. Pero, sobre todo, el aumento progresivo de cesáreas anteriores en mujeres gran multíparas dificulta bajar este indicador a los niveles recomendados(1). La proporción de nacimientos por cesáreas en todo el mundo ha aumentado significativamente en los últimos 35 años. Solo en Estados Unidos la tasa de cesáreas aumentó del 5\%, en 1970, al 31,8\%, en 2016, o sea un aumento del $26,8 \%$. En el África subsahariana el cambio en las tasas de los últimos 24 años fue del 2,3\% en 1990. Mientras que la tasa registrada en 2014 fue del 3,5\%. En la región de América Latina y el Caribe, en el mismo periodo, se observó un aumento importante: del $22,8 \%$ al $42,2 \%$. Un total de 850.000 habrían sido innecesarias(2).
Algunos estudios muestran una correlación importante entre el PIB per cápita y el aumento del número de cesáreas; se reportan diferencias entre hospitales públicos y privados. En hospitales privados de Brasil y Argentina este procedimiento puede alcanzar el $90 \%$ y el $76 \%$ en el caso de Chile(3). El último informe de la OECD-2017, compuesto por 35 países miembros, afirma que los países con tasas bajas siguen siendo los países nórdicos, Israel y los países bajos, con un 15\% a $17 \%$. Mientras tanto, Turquía reporta una tasa del 53\%, México, 46\% y Chile 46\%(4) (figura 1).

Otros estudios sugieren que las condiciones preexistentes, como la obesidad y el aumento de la edad materna, son consideradas por los obstetras como factores que podrían ser responsables del aumento de este procedimiento. También se considera como factor la solicitud de las madres relacionadas con temor al trabajo de parto, preocupación por compromiso del bienestar maternofetal, disfunción sexual, antecedentes de malas experiencias, autonomía de la mujer y relación médico paciente cada vez más horizontal(5). El aumento de las tasas de cesáreas por sí sola representa un proceso de desigualdades a nivel mundial, siendo una preocupación para la gestión de la salud y las políticas públicas(6,7). Del mismo modo, la Federación Internacional de Ginecología y Obstetricia (FIGO) y el Colegio Americano

Figura 1: Comparison of C-sections rates among OECD countries, 2013-20

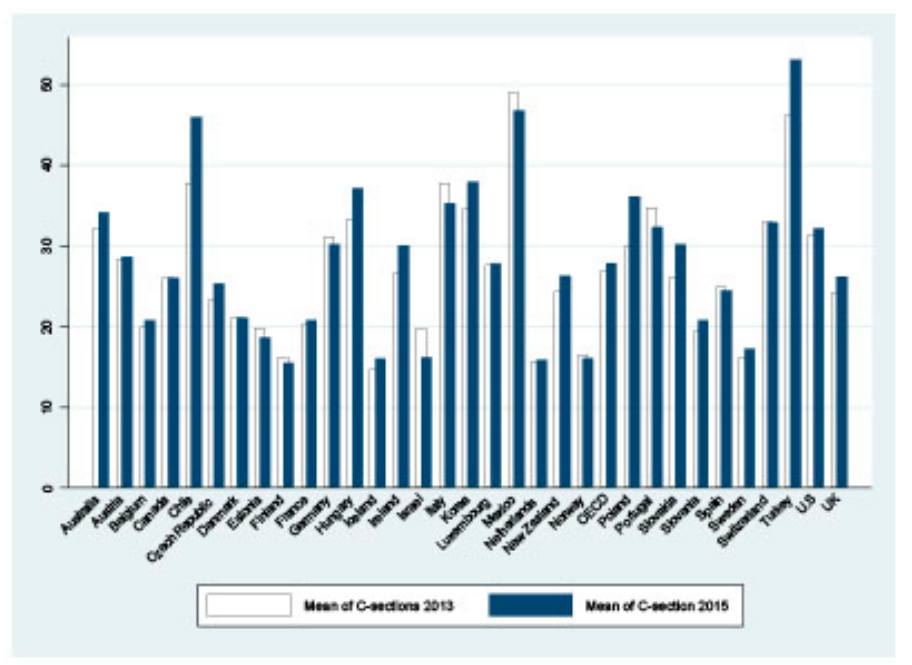

Fuente: Own elaboration from OECD Health Statistics 2017. 
de Obstetras y Ginecólogos (ACOG) establecen que la realización de cesáreas por razones no médicas es éticamente injustificada y no implica beneficios adicionales para la madre o el recién nacido(8) (tabla 1). Hoy existe suficiente evidencia que afirma que, debido a las cesáreas, las estancias hospitalarias son más extensas, los problemas respiratorios en el recién nacido son más frecuentes, con mayor probabilidad de complicaciones posteriores para la mujer, como, ruptura uterina, problemas de implantación de placenta y riesgo de realizar histerectomías $(9,10)$.

\section{Antecedentes del problema}

Se afirma que un factor importante es el aumento en la preferencia de las mujeres por las cesáreas, y que esto se debe a experiencias negativas durante el parto natural. Por otro lado, para el equipo de salud, las cesáreas programadas tienen algunos beneficios: reduce el tiempo de parto, no hay nece- sidad de controlar el trabajo de parto ni requiere inducirlo. Esto implica mayor eficiencia temporal y mayor capacidad de resolución, con mayor control del procedimiento. Sin embargo, es necesario tener presente que la eficiencia en tiempo no es directamente proporcional con la eficiencia en los procesos. En un estudio de seguimiento en el posparto a un total de 1.506 mujeres, entre 2007 y 2008, tratadas en tres hospitales en el centro de Suecia, se les preguntó sobre su preferencia. El $9,8 \%$ indicó que preferiría una cesárea la próxima vez. Los factores que influyeron para esta decisión fueron sentimientos de ambivalencia durante el embarazo, experiencias negativas previas de parto, miedo al parto, cesárea como opción segura y falta de información en los controles prenatales(11).

En Irán, durante 2013, se reportaron 42\% de cesáreas en hospitales públicos y $90 \%$ en hospitales privados. Los resultados mostraron cuatro causas principales por las cuales las mujeres iraníes pre-

Tabla 1: Indicaciones de Cesárea según causas fetales, ovulares y maternas.

\begin{tabular}{|l|l|}
\hline $\begin{array}{l}\text { SEGÚN } \\
\text { CAUSA }\end{array}$ & DESCRIPCIÓN \\
\hline FETALES & $\begin{array}{l}\text { Presentación distócica por lo que el parto vaginal no es posible: Tronco o podálica. } \\
\text { Sufrimiento fetal agudo: dilatación cervical incompleta, grado de encajamiento de la } \\
\text { presentación insuficiente. }\end{array}$ \\
& $\begin{array}{l}\text { Macrosomía fetal: Fetos }>4.500 \text { gramos } \\
\text { Mujer con Diabetes pregestacional y un peso fetal estimado entre } 4.000 \text { y } 4.500 \text { g, para } \\
\text { evitar complicaciones traumáticas en el parto }\end{array}$ \\
\hline \multirow{5}{*}{ OVULAR } & $\begin{array}{l}\text { Placenta previa: Completa: La placenta cubre toda la abertura cervical. } \\
\text { Desprendimiento prematuro de la placenta normoinserta (emergencia por hipoxia y muerte fetal } \\
\text { y posible hematomas retroplacentrios uterinos "Couvelaire") }\end{array}$ \\
& $\begin{array}{l}\text { Procidencia de cordón: Descenso del cordón umbilical a través del cuello uterino (Emergencia } \\
\text { que puede provocar hipoxia fetal). } \\
\text { Embarazos múltiples o gemelares: Indicaciones absolutas de cesárea son el embarazo } \\
\text { monoamniótico, gemelos pagos e independiente de la corionicidad, la presentación no cefálica } \\
\text { del primer gemelar. Indicación relativa serían la presentación cefálica del gemelo uno con } \\
\text { presentación no cefálica del gemelo dos. }\end{array}$ \\
\hline MATERNAS & $\begin{array}{l}\text { Mujer con antecedente de dos o más cesáreas por riesgo de rotura uterina } \\
\text { Cesárea anterior: razones obstétricas y/o con patologías maternas asociadas } \\
\text { Antecedentes de cirugía vaginal previa: Plastía vaginal } \\
\text { Transmisión vertical de infecciones maternas: VIH (+) y Hepatitis C. } \\
\text { Las mujeres con infección primaria de virus herpes simple genital (VHS), durante el tercer } \\
\text { trimestre de gestación. }\end{array}$ \\
\hline
\end{tabular}

Fuente: Guía Perinatal 2015: Programa Nacional Salud de la Mujer. Ministerio de Salud. Gobierno de Chile 
ferían las cesáreas: creencias personales, miedo debido a experiencias previas, normas y valores culturales, evitar el dolor, prevenir complicaciones y protección de la anatomía genital(12).

En Chile, entre 2006 y 2012, el promedio de cesáreas en el país fue del 37\%; sin embargo, cuando la información se desagrega al nivel local, algunas poblaciones reportaron hasta un $60 \%$. Un estudio sobre las percepciones y actitudes de 180 mujeres tratadas en clínicas privadas reportó una tasa de cesárea del $60 \%$, y en hospitales públicos se aproximó al 22\%. Los resultados sugirieron que el $77,8 \%$ de las mujeres habría preferido un parto vaginal, mientras que solo el 9,4\% habría preferido la cesárea. No se encontraron diferencias significativas entre la paridad, los ingresos o la educación(13). Siempre en Chile, un estudio realizado en 2014 entrevistó a 396 mujeres de la Región Metropolitana con al menos una cesárea en los últimos 10 años. Solo el 6,6\% pidió la primera cesárea y la segunda cesárea fue el 9,9\%(14).

Lo expuesto invita a reflexionar sobre lo idóneo de la cesárea electiva sin indicación médica o emergencia, y a veces sin ser una preferencia real. Esto genera varias preguntas: las mujeres que se somete a una cesárea, ¿están debidamente informadas del procedimiento? ¿Será adecuada la información? ¿Es comprensible? ¿Entenderán los riesgos y complicaciones?

\section{Consentimiento informado}

El consentimiento informado en la atención médica está legalmente registrado por primera vez en el "caso Salgo", que tiene lugar en los Estados Unidos en 1957, en el que se discutió si un paciente debe o no conocer toda la verdad sobre sus condiciones de salud(15). En Chile, en abril del 2012, se dicta la Ley No 20.584, que declara los derechos y deberes de los pacientes mediante la regulación del consentimiento informado, proporcionando autonomía para decidir sobre un procedimiento médico(15-17). En el artículo 14 se reconoce el derecho de los pacientes al consentimiento informado: "Toda persona tiene derecho a aceptar o no someterse a cualquier procedimiento o tratamiento relacionado con su atención médica, dentro de las limitaciones establecidas en el artículo 16", haciendo esto referencia a la prohibición de aceleración artificial de la muerte, la eutanasia y el suicidio asistido. La ley añade que el consentimiento debe ejercerse libre y voluntariamente, expresado e informado. Para que sea eficaz, el profesional sanitario debe proporcionar al paciente información oportuna, adecuada, suficiente y comprensible sobre el estado de salud, el posible diagnóstico, el procedimiento quirúrgico a realizar, el procedimiento anestésico, las alternativas de tratamiento disponibles, los riesgos de ambos procedimientos, el resultado esperado y el proceso postoperatorio predecible(17). Es fundamental destacar que, aunque la ley establece el consentimiento como un "proceso de información", sea cual sea el procedimiento para realizar, en una cesárea resulta paradójico que la información que llega a la usuaria en un alto porcentaje no es oportuna, suficiente ni comprensible; muy por el contrario, el proceso no es más que la firma de un documento a adjuntar en la ficha clínica. Además, en Chile no existen formularios de consentimiento informado específicos para cada procedimiento. La Superintendencia de Salud, en su página web, sugiere la adaptación de los formularios de consentimiento desarrollados en España por el Consejo de Andalucía y Valencia(18).

\section{El problema bajo el enfoque de la bioética}

En el escenario mundial se conoce el Código de Núremberg, la Declaración Helsinki, el Informe Belmont, las Pautas Internacionales de las Ciencias Médicas (CIOMS). Sin embargo, estas pautas solo abordan aspectos éticos en investigación clínica en seres humanos. Durante 1979, Tom Beauchamp, miembro de la Comisión Nacional para la Protección de los Sujetos Humanos de Investigación Biomédica y del Comportamiento, y James Childress, reformularon estos principios para ser aplicados a la ética del cuidado de la salud, colocando la autonomía en primer lugar, estableciendo así la posición de la corriente principialista, compuesta por la no maleficencia, la beneficencia, la autonomía y la justicia(19). Los escenarios anteriores sugieren debatir sobre la naturaleza de la autonomía en el parto de las mujeres. Este debate se centraría en lograr un equilibrio entre quienes defienden la autonomía de la mujer en el proceso de parto y el principio de beneficencia y no maleficencia. 
En este sentido, la cesárea profiláctica fue citada por primera vez por Nubiolo Espino en 1950, término que utilizó para describir los alcances futuros de la intervención medicalizada de los partos(20). Pero una cesárea, así como un parto vaginal bien indicado y justificado, deberían terminar con buenos resultados. Por otro lado, la asistencia clínica y la salud pública usan cada vez más medios coercitivos y paternalistas. El médico asume la toma de decisiones como un deber, en el lugar de la mujer, y el paternalismo estatal se compromete a garantizar la mejor atención, incluso utilizando medios coercitivos para proteger a las personas de sí mismas. Esto es útil para casos como tabaco, alcohol, drogas, entre otros, pero hay otros aspectos en la atención médica que requerirán que la persona sea informada y tenga el entendimiento suficiente para asumir una decisión del procedimiento que le será aplicado(21). Por lo tanto, ¡es ético realizar una cesárea electiva en una mujer con un embarazo normal a término, sin factores de riesgo o complicaciones? ¿Cuál sería la respuesta más apropiada del obstetra/ginecólogo para la paciente que solicita una cesárea programada sin indicación médica?

\section{El dilema ético}

Se propone analizar el problema y su dilema ético subyacente a través del enfoque consecuencialista (utilitarismo), la teoría kantiana (ética), la ética de la virtud y la teoría feminista (la ética del cuidado y la ética del feminismo liberal). Las dos primeras teorías morales son punto de partida de cualquier discusión. La posición consecuencialista sostiene que el propósito de una acción es la base para cualquier apreciación moral $(22,23)$. Este enfoque propone que todas las obligaciones morales se derivan del principio de utilidad, donde la acción moralmente correcta conduce a buenas consecuencias. Por lo tanto, el debate no se centraría en realizar o no una cesárea, sino en el resultado. En este caso, una mujer y nińos sanos, sin complicaciones, harían moralmente tolerable hacer el procedimiento. La visión utilitaria de Jeremy Bentham (1748-1832) propuso que cualquier obligación moral deriva en última instancia del principio de utilidad, que considera que, dentro del grupo de acciones alternativas, nos vemos obligados a elegir una que conlleve mayor beneficio al mayor número de personas; sin embargo, en medicina cada caso es particular y no siempre el beneficio es numeroso. John Stuart Mill (1806-1873) desarrolló dos posturas utilitarias. La primera considera que una acción es apropiada $\mathrm{si}$, entre las diferentes alternativas, hay una diferencia entre las consecuencias buenas y malas. La segunda considera que una acción determinada es obligatoria si el resto de las posibles acciones generan una diferencia inferior entre sus buenas y malas consecuencias. Sin embargo, no siempre es posible tener los recursos humanos y técnicos a disposición para una adecuada respuesta y, por ello, el principio de utilidad se limita por el principio de posibilidad y de oportunidad(24-26).

En el caso de la teoría kantiana, la buena voluntad no es buena por los efectos de sus acciones, sino solo por su voluntad misma. Por ejemplo, un individuo X (no fumador) y un individuo Y (fumador) entran en un establecimiento libre de humo. El individuo X no fuma, por lo tanto, actúa de acuerdo con el deber porque no fumará ya que es un no fumador; por el contrario, el individuo Y (fumador), se abstendrá de fumar. Solo en este último caso hay una acción con valor moral en el sentido kantiano. Esto se debe al hecho de que la acción no está motivada por una inclinación, sino por el deber al respeto a la ley. En el caso de nuestra discusión, el énfasis estaría en las motivaciones de una cesárea. Por lo tanto, mientras exista la posibilidad de una acción o interés subyacente diferente a lo establecido en los protocolos para una cesárea, esta no sería moralmente tolerable $(26,27)$. Respecto de la autonomía, según Beauchamp y Childress, el individuo autónomo es aquel que actúa libremente de acuerdo con un plan auto elegido. A este respecto, se afirma que existen dos condiciones esenciales: la libertad y el albedrío; la primera se entiende como la independencia de controlar las influencias y la segunda como la capacidad de acción intencional. Esto teniendo en cuenta que, en teoría, una persona autónoma puede ser identificada por los siguientes rasgos: excepcionalmente auténtica, poseída por sí mismo, consistente, independiente, autogobernada, resistente al control por parte de las autoridades y fuente original de valores, creencias y planes de vida personales, conducentes a tomar las mejores decisiones para el camino que quiere llevar(28). 
Por otro lado, la ética de la virtud, postulada por MacIntyre, también conocida como neo aristotelismo, sostiene que no es posible formular una moralidad universal, o filosofía moral independiente de las circunstancias o contextos sociales, y que para hacer filosofía moral es necesario integrar la antropología, la historia y la sociología. Esto se debe a la creencia que cualquier teoría moral es una respuesta a ciertas necesidades históricas de la sociedad. Basándonos en esto debemos preguntarnos acerca de la posibilidad de encontrar diferentes tipos de ética, enmarcadas en contextos específicos dentro de diferentes comunidades en las que se desarrollan diferentes ideales; por lo tanto, se desarrollan diferentes éticas de la virtud. Esto implicaría una posición de características individualistas, lo que sería bueno para una comunidad no lo sería para otra. Si volvemos al estudio iraní mencionado, los factores que determinaron la preferencia por la cesárea relacionada con valores culturales se enmarcarían en la ética de la virtud. Sin embargo, se imponen nuevas tecnologías médicas en sentido contrario a estas culturas(28).

Siguiendo con la reflexión ética, se consideró la teoría feminista (ética del cuidado y feminismo liberal). En este sentido, se ha propuesto aplicar la ética de la atención a los problemas morales en el ámbito de la salud, como por ejemplo la reproducción asistida, la genética y el aborto, entre otros. La ética del cuidado sostiene que es posible dar cuenta de la naturaleza de la moralidad y formula la ética normativa a partir de valores femeninos, como la empatía, la compasión, la conciencia, el compromiso, el cuidado mutuo y las responsabilidades en las relaciones de reproducción y supervivencia(29). En la actualidad es importante distinguir la ética de la atención arraigada en la teoría feminista, que propone analizar y criticar cualquier forma de injusticia de género (discriminación, desigualdad, exclusión), y que fortalece la autonomía de las mujeres en la toma de decisiones sobre su salud.

Los primeros intentos de desarrollar una filosofía moral desde una perspectiva y valores específicos de la feminidad están cerca de la tradición humanista(30). El segundo intento surge de la reacción conservadora, conocida por esta corriente como "androcentrismo", y el patriarcado, que se basa en los valores instalados por Rousseau, quien define la feminidad esencialista como coherente con los valores mantenidos en el contrato social del siglo XVIII, que se rompe con el surgimiento del feminismo(31). La ética del cuidado pone de relieve las relaciones morales y de responsabilidad marcadas por la preocupación transversal por otras personas. Por esta razón, esta ética se vincula básicamente en el contexto de las relaciones interpersonales, en las que es posible sentir empatía por el otro. Sobre esta base, las decisiones morales dependerán de las relaciones interpersonales y de los contextos específicos en los que se vive la experiencia. Por lo tanto, es difícil establecer posiciones que ayuden a tomar medidas ante un dilema ético aplicable para todos(32). El feminismo liberal, tiene sus raíces en los principios liberales, hace hincapié en la defensa de los derechos humanos inalienables para las mujeres y promueve la autonomía en todas sus dimensiones. En salud, la reivindicación se centra en los derechos humanos, especialmente en los derechos sexuales y reproductivos como argumento moral para proteger a las mujeres(32). Sin embargo, no hay duda de que la ley en sí misma no responde a las preocupaciones morales que podrían surgir en situaciones relacionadas con su salud, su cuerpo y su vida. El mero ejercicio de los derechos no garantiza la acción moral. Debe tenerse en cuenta que tomar una decisión puede tener impacto en el desarrollo de complicaciones médicas después de una intervención. La mujer puede tener derecho a decidir sobre un procedimiento de cesárea y no un parto natural, o viceversa, pero el personal de salud tratante está obligado a indicar los pros y los contras, así como las complicaciones posteriores a cualquier intervención y asegurarse que la mujer las ha comprendido(33).

\section{Conclusiones}

Debe reconocerse que el dilema ético moral en el contexto de un elevado número de cesáreas que se realiza hoy en día, está inmerso en múltiples factores individuales, sociales, culturales, entre otros determinantes de la salud que deben ser considerados en la toma de decisiones. No siempre es posible contar con todos los recursos

Se debe fortalecer el deseo de la paciente informada y el médico debe seguir los protocolos tomando decisiones sobre la base de la mejor evidencia 
disponible y una mejor preparación de las mujeres en la atención prenatal, de modo que, cuando llegue el momento del parto, la decisión se tome con la mejor información disponible. Por otro lado, no siempre se cuenta con los mejores recursos para diagnóstico y tratamiento, por lo que las decisiones están delimitadas por las posibilidades y oportunidades. Esto hace necesario reconocer la condición de sujeto de las mujeres en el proceso de atención, lo que implica el respeto de su autonomía, capacidad de comprensión, posibilidad de deliberación y toma de decisión.
Por otra parte, aunque la OMS/OPS ha establecido una tasa aceptable de cesáreas como procedimientos, todavía se observan tasas muy altas. Desde la salud pública se sugiere estudiar el parámetro de "tasas aceptables", ya que esta puede forzar la realización de partos vaginales por tratar de alcanzar tasas aceptables de cesáreas. El parto es un evento natural y se debe basar en el respeto de los procesos biológicos. Por lo tanto, las cesáreas deben ser vistas como un medio para este proceso natural, pero en presencia de complicaciones e indicaciones respaldadas con la mejor evidencia disponible, siendo lícito como cualquier procedimiento en salud.

\section{Referencias}

1. Organización Mundial de la Salud. Estadísticas Sanitarias Mundiales 2015. Disponible en http://www.bvs.hn/docum/ ops/WHO-HIS_HSI_15.1_eng.pdf.

2. Beltrán AP, Ye J, Moller AB, Zhang J, G-lmezoglu AM, Torloni MR La tendencia creciente en las tasas de cesáreas: estimaciones mundiales, regionales y nacionales: 1990-2014. PLoS Uno 2016; 11(2): 1-12.

3. Mariani GL, Vain NE. La incidencia y el impacto de la cesárea pre-trabajo no médicamente indicada en América Latina. Seminarios en Medicina Fetal y Neonatal 2019; 24: 11-17.

4. Organización para la Cooperación y el Desarrollo Económicos. Salud de un vistazo. París: OECD Publishing; 2017. Disponible en https://www.oecd-ilibrary.org/social-issues-migration-health/health-at-a-glance-2017_health_glance2017-en Google Scholar

5. Vergara F, Abel F, Álvarez D, Coll C, Cuadrado C, González J, Martínez JM, Mateu S, Zamarriego J. Ethical considerations about caesarean section. SEGO consensus document. Prog Obstet Ginecol 2001; 44: 46-55.

6. Colegio Americano de Obstetras y Ginecólogos. Prevención segura del parto por cesárea primaria. Consenso en la atención obstétrica 2014; 123: 693-711.

7. Federación Internacional de Ginecología y Obstetricia. Directrices éticas sobre la reproducción humana y la salud de las mujeres. Revista Internacional de Ginecología y Obstetricia 1999; 64: 317-322.

8. Colegio Americano de Obstetras y Ginecólogos. Dictamen del Comité no 559: Parto por cesárea a petición materna Obstet Gynecol 2013; 121(4): 904-907.

9. Organización Mundial de la Salud. Declaración de la OMS sobre la tasa de cesárea. Departamento de Salud Reproductiva e Investigación; 2015. Disponible en http://apps.who.int/iris/bitstream/10665/161444/1/WHO_RHR_15.02_spa. pdf?ua $=1$

10. Vallejos A, Espino S, López M, Duran L. ¿Prefieren las mujeres cesáreas? Perinatol Reprod Hum 2016; 1330(3): 127129.

11. Karlstrom A, Nystedt A, Johansson M, Hildingsson I. Detrás del mito, pocas mujeres prefieren la cesárea en ausencia de factores médicos u obstétricos. Partería (2011; 27(5): 620-7.

12. Latifnejad-Roudsari R, Zakerihamidi M, Merghati-Khoei E, Kazemnejad A. Percepciones culturales y preferencias de las mujeres iraníes con respecto al parto por cesárea. Irán J Nurs Partewifery Res 2014; 19: S28-36.

13. Sadler M, Leiva G. Percepciones y prácticas en la cesárea. FONIS SA13I20259, Universidad de Chile; 2016. Disponible en https://www.researchgate.net/publication/290433891_Percepciones_y_practicas_sobre_la_operacion_cesarea_FONIS_SA13I20259

14. Angeja AC, Washington AE, Vargas JE, Gomez R, Rojas I, Caughey AB. Preferencias de las mujeres chilenas con respecto al modo de parto: ¿¿cuáles prefieren y por qué? BJOG 2006; 113: 253-258.

15. Escobar MT, Carrera CA. Percepción del consentimiento informado en pacientes de cuatro áreas diferentes de atención médica en Bogotá, Colombia. Revista Latinoamericana de Bioética 2015; 15: 14-2.

16. Salgado VR. Afirma que existe una "obligación de indemnizar cuando se superaron los límites del consentimiento otorgado por 
Tendencia de la cesárea: Una reflexión desde la ética - Jairo Javier Vanegas López, Amaya Pávez Lizárraga, Patricia Muñoz Ojeda

el paciente o se ocultó una prohibición de él". Leland Stanford Jr University Board of Trusted; 1975. Disponible en http:// www.institutodebioetica.org/casosbioetica).

17. Figueroa R. Consentimiento informado en la nueva ley sobre los derechos de los pacientes. Rev. méd Chile 2012; 140: 1347-1351.

18. Ministerio de Salud. Formulario de consentimiento informado. 2019 Disponible en http://www.supersalud.gob.cl/observatorio/575/w3-article-7651.html.

19. Beauchamp TL, Childress JF. Principios de Ética Biomédica. Nueva York/Oxford: Oxford University Press; 1999.

20. Carrera JM. El feto como paciente: nuevos retos. Ginecología y Obstetricia Clínica 2003; 4: 126-127.

21. Cohen D. Responsabilidad y libertad. Hispanic American Philosophy Magazine 2003; 35(105): 103-110.

22. Kishore RR. Human organs, scarcities, and sale: morality revisited. J Med Ethics 2005; 31: 262-265.

23. Kunin JD. The search for organs: halachic perspectives on altruistic giving and the selling of organs. J. Med. Ethics 2005; 31: 269-272.

24. Taylor S. Autonomía, opciones de restricción y ventas de órganos. Revista de Medicina y Filosofía 2009; 34: 632-648.

25. Carrasco M. Algunas objeciones de consecuencialismo ético. Universalismo, Relativismo, pluralismo. Thémat 2001; 27: 155-163.

26. Cejudo R. Deontología y consecuencialismo: un enfoque informativo. Crítica, una revista de flosofía hispano-estadounidense 2010; 126: 3-24.

27. Zamora A. Kantian deontology. Rev. filosofía Univ. Costa Rica 2005; XLIII(108): 147-151.

28. Siurana J. Los principios de la bioética y la aparición de la bioética intercultural. Veritas 2010; 22: 121-157.

29. Gilligan C. En una voz diferente. Teoría psicológica y desarrollo de la mujer. Harvard University Press; 2016.

30. Ortiz G. La ética feminista, la ética femenina y el aborto. Debate Feminista 2014; 49(34): 70-93.

31. Friedan, B. La mistica de la feminidad. Madrid, España: Editorial Cátedra; 2016.

32. Sañudo M, Vásquez J. Aproximación ética y racional de la operación por cesárea. Realidad y Reflexión 2016; 16: 82-91.

33. Correa L. La ética del cuidado feminista y la bioética personal. Prest Bioét 2008; 30: 53-61.

Recibido: 19 de marzo de 2021

Aceptado: 5 de abril de 2021 